\title{
Digitally Enhanced Heterodyne Interferometry
} This design mitigates cyclic error and improves measurement sensitivity.

\section{NASA's Jet Propulsion Laboratory, Pasadena, California}

Spurious interference limits the performance of many interferometric measurements. Digitally enhanced interferometry (DEI) improves measurement sensitivity by augmenting conventional heterodyne interferometry with pseudo-random noise (PRN) code phase modulation. DEI effectively changes the measurement problem from one of hardware (optics, electronics), which may deteriorate over time, to one of software (modulation, digital signal processing), which does not. DEI isolates interferometric signals based on their delay. Interferometric signals are effectively time-tagged by phase-modulating the laser source with a PRN code. DEI improves measurement sensitivity by exploiting the autocorrelation properties of the PRN to isolate only the signal of interest and reject spurious interference. The properties of the PRN code determine the degree of isolation.

Heterodyne interferometers are used for metrology to measure the distance to a remote mirror, usually with several intermediate surfaces for steering and beam-shaping. These surfaces, while necessary, can degrade the measurement. A digitally enhanced heterodyne interferometer makes the same measurement, with the primary difference being the addition of an electro-optic modulator that adds zero or $\pi$ phase shift onto the measurement beam before it goes out to the measurement surface. The electronics system has an additional front end that demodulates the PRN code before the phase measurement (see figure).

The PRN code used is a maximallength sequence. This sequence has the property that, when correlated with itself, is one and, when correlated with a delayed version of itself, the correlation can be as low as $1 / N$, where $N$ is the length of the code sequence (chip length). The pseudo-random code appears as white noise on the photodetector. The heterodyne signal appears only after the signal is demodulated with the proper delay by the electronics. Cyclic

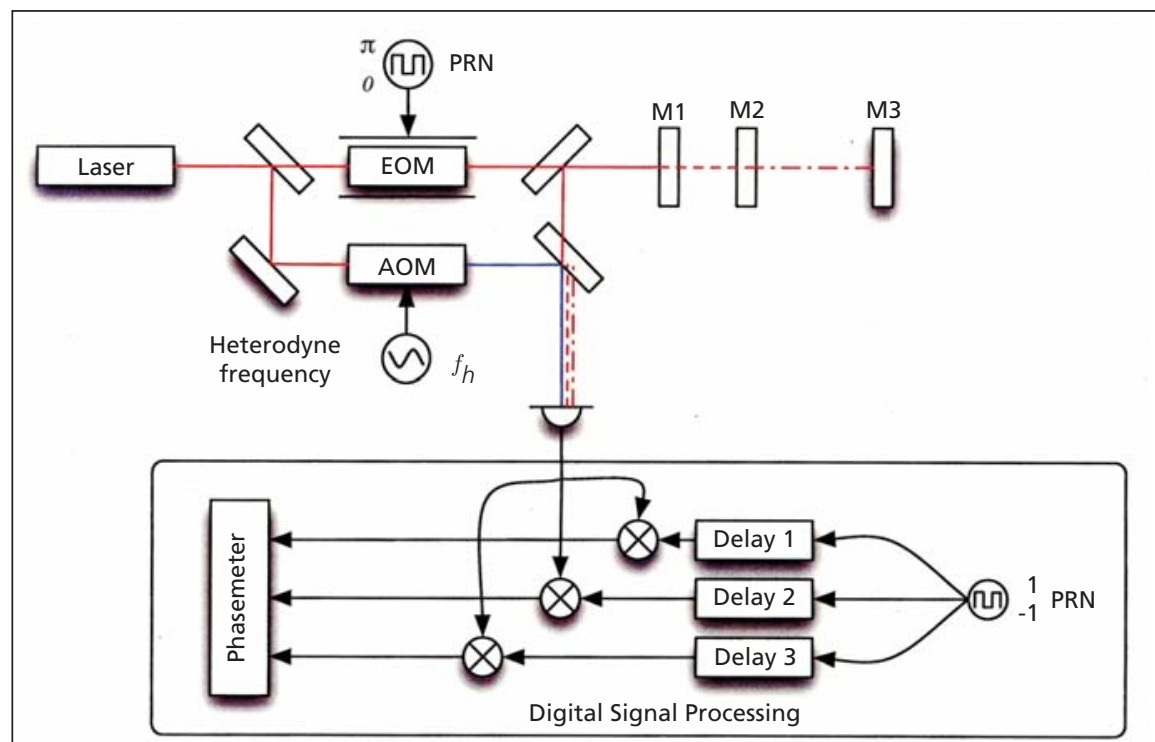

A Digitally Enhanced Heterodyne measurement system includes an electro-optic modulator (EOM) and an additional front end on the phasemeter that demodulates the PRN code before the phase is measured for the metrology.

errors can be suppressed up to $1 / N$ by adjusting the delay of the PRN code in the front end of the phasemeter to measure the desired signal and reject spurious interference. By adjusting the delay properly, signals from the spurious reflections can also be analyzed.

PRN code modulation is conceptually similar to code division multiplexing, which is used in communications for spread spectrum signals. The technology for this is widespread and mature. PRN codes are commonly used in metrology, e.g. lidar systems, by amplitude modulating a laser with a PRN code and performing range measurements based on the code. The sensitivity of these measurements is determined by the characteristic wavelength of the PRN code. In contrast, digitally enhanced interferometry sensitivity is determined by the optical wavelength, not the code, and is several orders of magnitude more sensitive.

Another benefit of delay-based signal isolation is the ability to measure multiple optical components with a single metrology system. This multiplexing capability significantly simplifies measurements of multiple components, such as displacement monitoring of a train of optics or segments of a multi-mirror telescope. This design's immunity to scattered light and electronic interference will allow measurements to approach the fundamental limits of shot noise and electronic noise, allowing for laboratory-class performance in real-world environments.

This work was done by Daniel Shaddock, Brent Ware, Oliver Lay, and Serge Dubovitsky of Caltech for NASA's Jet Propulsion Laboratory.

In accordance with Public Law 96-517, the contractor has elected to retain title to this invention. Inquiries concerning rights for its commercial use should be addressed to:

Innovative Technology Assets Management JPL

Mail Stop 202-233

4800 Oak Grove Drive

Pasadena, CA 91109-8099

E-mail: iaoffice@jpl.nasa.gov

Refer to NPO-45188, volume and number of this NASA Tech Briefs issue, and the page number. 